\title{
Antitumor Potential of Fibulin-5 in Breast Cancer Cells Depends on Its RGD Cell Adhesion Motif
}

\author{
Yamina Mohamedia Tania Fontanila,b,c Teresa Cobo ${ }^{c, d} \quad$ José A. Vega ${ }^{e, f}$ \\ Juan Luis Cobo ${ }^{\mathrm{e}, g}$ Marcos Pérez-Basterrechea ${ }^{\mathrm{h}}$ Juan Cobo ${ }^{\mathrm{c}, \mathrm{d}}$ \\ Álvaro J. Obayab,i Santiago Cal ${ }^{a, b}$
}

aDepartamento de Bioquímica y Biología Molecular, Universidad de Oviedo, Asturias, Spain, 'Instituto Universitario de Oncología, IUOPA, Universidad de Oviedo, Asturias, Spain, Instituto Asturiano de Odontología, Oviedo, Asturias, Spain, 'Departamento de Cirugía y Especialidades Médico-Quirúrgicas, Universidad de Oviedo, Asturias, Spain, eDepartamento de Morfología y Biología Celular, Facultad de Medicina, Universidad de Oviedo, Asturias, Spain, ${ }^{\mathrm{f} F a c u l t a d}$ de Ciencias de la Salud, Universidad Autónoma de Chile, Santiago, Chile, ${ }^{9}$ Servicio de Cirugía Maxilofacial, Hospital Universitario Central de Asturias (HUCA), Asturias, Spain, hUnidad de Terapia Celular y Medicina Regenerativa, Servicio de Hematología y Hemoterapia, Hospital Universitario Central de Asturias, Asturias, Spain, 'Departamento de Biología Funcional, Area de Fisiología, Universidad de Oviedo, Asturias, Spain

\section{Key Words}

Fibulin • Breast cancer • Tumor microenvironment • RGD motif

\begin{abstract}
Background/Aims: Different components of the tumor microenvironment can be either tumor-promoting or tumor-suppressive agents depending on factors which are not fully understood. Fibulins are components of the extracellular matrix from different tissues and constitute a clear example of this dual function. In fact, fibulins may either support tumor growth or abolish progression of malignant cells depending on the crosstalk between tumor cells and their surrounding stroma through mechanisms that remain to be elucidated. Among all fibulins, fibulin-5 contains a particular structural hallmark which consists in the presence of a RGD motif within its architecture. Previous reports have highlighted the importance of the interaction of this motif with integrins, and not only in normal functions but also in a tumor context. Methods: Site-Directed Mutagenesis technique was employed to introduce the change RGD to RGE (RGD-to-RGE) within Fbln5 cDNA sequence. Cell proliferation was measured using the MTT assay or by counting Ki-67 positive cell nuclei. Cell adhesion was analysed using culture plates coated with different extracellular matrix components. Cell invasion was evaluated using 24-well Matrigel-coated invasion chambers, and mammosphere formation was monitored using ultralow attachment culture plates. BALB/C mice were employed to induce subcutaneous tumors. Results: The RGD-to-RGE change alters the capacity of breast cancer cells to adhere to different extracellular matrix proteins as well as

\begin{tabular}{ll}
\hline Santiago Cal & Departamento de Bioquímica y Biología Molecular, Facultad de Medicina, \\
and Álvaro J. Obaya & Universidad de Oviedo, 33006 Oviedo (Spain) \\
& Tel. +34 985106282, E-Mail santical@uniovi.es; ajobaya@uniovi.es
\end{tabular}
\end{abstract}




\section{Cellular Physiology Cell Physiol Biochem 2019;53:87-100 \\ \begin{tabular}{l|l} 
and Biochemistry Published online: 18 June 2019 & $\begin{array}{l}\text { DO } 2019 \text { The Author(s). Published by } \\
\text { Cell Physiol Biochem Press GmbH\&Co. KG }\end{array}$ \\
\hline
\end{tabular} \\ Mohamedi et al.: Fibulin-5 RGD Motif in Breast Cancer Cells}

to $\alpha_{v} \beta_{3}$ and $\alpha_{5} \beta_{1}$ integrins, and promotes protumor effects using different cell-based assays. Moreover, 4T1 cells, a mouse breast cancer cell line model, shows an increased capacity to generate tumors when exogenously expresses fibulin-5 with a RGD-to-RGE change, and such capacity is similar to that shown for 4T1 cells with an interfered Fbln 5 gene. Conclusion: These data highlight the importance of the RGD motif of fibulin-5 to induce antitumor effects and provide new insights into the involvement of fibulins in tumor processes.

(C) 2019 The Author(s). Published by Cell Physiol Biochem Press GmbH\&Co. KG

\section{Introduction}

Acellular components of the cell microenvironment form a structural framework to give support to cells and tissues. However, this intricate network of complex molecules and sequestered growth factors, known as extracellular matrix (ECM), not only forms an architectural scaffold for cells but also regulates cellular activities that influence processes such as differentiation, proliferation or migration [1]. Functional significance of ECM is illustrated by the number of anomalies associated to its components which lead to the development of pathological conditions, including cancer [2]. In fact, ECM is highly disorganized in tumor processes and can contribute to promote growth and dissemination of malignant cells [3]. However, different ECM components can act as protumor or antitumor factors through mechanisms not fully understood and fibulins exemplify this dual function in tumorigenesis $[4,5]$. Originally identified as components of elastic fibers, fibulins play key roles in a wide range of biological processes related to tissue homeostasis and remodeling; and their biological importance is highlighted through the severe deficiencies shown by the mouse models lacking a particular fibulin gene [6]. In relation to cancer, a growing number of studies have revealed that effects of fibulins are complex and variable dependent on different factors [5].

Fibulin-5, also known as EVEC and DANCE, constitutes a clear example of the importance of fibulins in those processes. This 66-kDa glycosylated protein contains different motifs within its structure involved in the interaction with other ECM proteins [7, 8]. Generation of the mouse deficient in fibulin-5 coding gene, Fbln5, revealed its importance in biological functions [9, 10]. Although Fbln5-deficient mice survive to adulthood, they develop severe abnormalities derived from elastic fiber defects, including genital prolapse, emphysematous lungs and cutis laxa. Regarding to tumor processes, both protumor and antitumor roles have been associated to fibulin-5, which seem to be context-dependent [11]. For instance, levels of FBLN5 messenger RNA were found downregulated in a variety of human tumor samples [11]; and fibulin-5 inhibits invasion of bladder [12] and lung [13, 14] cancer cells through different mechanisms. These findings suggest a tumor-suppression function for fibulin-5. In stark contrast, fibulin-5 mediates lung metastasis in a rat model of renal cell carcinoma [15] and also induces nasopharyngeal carcinoma cell metastasis [16]. Furthermore, fibulin-5 is associated to EMT in breast [17] and cervical cancer cells [18], via TGF- $\beta$ and Nogo-B dependent-mechanisms respectively.

We have previously reported the tumor-suppressive effects of the exogenous fibulin-5 expression in breast cancer cells [19]. Taking into account that fibulin-5 is the only member of the fibulin family containing a RGD integrin binding motif [7], and the importance of this motif not only in normal physiological conditions but also in tumorigenesis [20], we wanted to investigate whether the RGD-to-RGE change could affect behavior of the murine 4T1 and the human MDA-MB-231 breast cancer cell lines. Our data show a potent protumor activity of cells expressing a recombinant fibulin- 5 containing this change, and provide new insights into the significance of fibulin- 5 in tumor processes. 


\section{Cellular Physiology Cell Physiol Biochem 2019;53:87-100 \\ $\begin{array}{ll}\text { and Biochemistry Published online: } 18 \text { June } 2019 & \begin{array}{l}\text { DOI: } 10.33594 / 000000123 \\ \text { Pell Physiol Biochem Press GmbH\&Co. KG }\end{array}\end{array}$ \\ Mohamedi et al.: Fibulin-5 RGD Motif in Breast Cancer Cells}

\section{Materials and Methods}

\section{Cells lines and cell culture conditions}

Human MDA-MB-231 and murine 4T1 breast cancer cell lines were kindly provided by Dr. Carlos López-Otín and Dra. Ana Gutiérrez-Fernández. (University of Oviedo, Spain). Cells were routinely maintained in Dulbecco's Modified Eagle's medium (DMEM) containing $10 \%$ fetal bovine serum, $50 \mu \mathrm{g} / \mathrm{mL}$ streptomycin and $100 \mathrm{U} / \mathrm{mL}$ penicillin (Life Technologies). For transfection experiments, vector containing the human full-length cDNA for fibulin-5 was kindly provided by Dr William P. Schiemann (Case Western Reserve University, Cleveland, USA). RNA interference kit for knocking down Fbln5 gene was purchased from Origene. Vectors were transfected into cells at $75 \%$ confluence using Lipofectamine reagent (Life Technologies) as recommended by the manufacturer. Cells transfected with an empty vector (control) or stably expressing FBLN5 (Fibulin5-WT) were selected with $500 \mu \mathrm{g} / \mathrm{mL} \mathrm{G418} \mathrm{(Sigma-Aldrich);} \mathrm{and} \mathrm{cells}$ with interfered FBLN5 gene (Fibulin-5KD cells) with $0.5 \mu \mathrm{g} / \mathrm{mL}$ puromycin.

\section{Mutagenesis}

The QuikChange II XL Site-Directed Mutagenesis Kit (Agilent) was employed to introduce the change RGD to RGE (RGD-RGE) within the Fbln5 sequence, following the manufacturer's instructions. Primers designed for mutagenesis were: FBLN5MUT-Fw $5^{\prime}$-GCCTGCCGAGGAGAGATGATGTGTGTTAAC-3' and FBLN5MUT-Rv $5^{\prime}$ - GTTAACACACATCATCTCTCCTCGGCAGGC $-3^{\prime}$, Vector containing the full-length cDNA for FBLN5 was employed as template. PCR conditions were as follows: $95^{\circ} \mathrm{C}, 1 \mathrm{~min}(1 \mathrm{cycle}) ; 95^{\circ} \mathrm{C}, 50 \mathrm{~s}, 66^{\circ}$, $50 \mathrm{~s}$ and $68^{\circ} 12 \mathrm{~min}$ ( $18 \mathrm{cycles}$ ); and $68^{\circ} \mathrm{C}, 7 \mathrm{~min}$ ( 1 cycle). PCR products were visualized in a $1.0 \%$ agarose gel and DNA sequence was verified before experimental use. Breast cancer cells expressing mutant FBLN5 (Fibulin5-RGE) were selected with $500 \mu \mathrm{g} / \mathrm{mL}$ G418 (Sigma-Aldrich) as described above.

\section{Western-blot and immunocytochemical analysis}

Cell extracts were resolved by 8 or 10 polyacrylamide gel electrophoresis, transferred to a PVDF membrane (Millipore) and subsequently probed with the indicated antibodies. The primary antibodies were anti-fibulin- 5 antibody from Origene or Santa Cruz Biotechnology; anti- $\beta$-actin and anti- $\alpha$-SMA antibodies from Sigma-Aldrich; anti-Ki-67, anti-E-cadherin, anti-N-cadherin and anti-vimentin from Santa Cruz Biotechnology. Immunoreactive proteins were visualized using HRP-peroxidase labeled anti-rabbit or anti-mouse secondary antibody and the ECL detection system (Pierce). For immunocytochemical analysis, 4T1 cells were fixed with $4 \%$ paraformaldehyde and then were blocked with $10 \%$ fetal bovine serum. To detect Ki-67, fixed cells were incubated overnight with the H-300 antibody from Santa Cruz Biotechnologies, followed by $1 \mathrm{~h}$ incubation with a secondary Alexa 546-conjugated antibody (Life Technologies). DAPI was added at $100 \mathrm{ng} / \mathrm{mL}$ to visualize DNA in cell nucleus. Images were obtained using a fluorescence microscope (Axiovert).

\section{Cell proliferation assay}

Cell proliferation was measured using the CellTiter 96 Non-radiactive Cell Proliferation Assay kit (Promega). MBA-MB-231 and 4T1 cells $\left(5 \times 10^{3} /\right.$ well) were seeded in 96-well plates and six replicates were performed per condition. Cell proliferation rates were determined on four (4T1) or five (MDA-MB-231) consecutive days using an automated microtiter plate reader Power Wave WS (BioTek). Additionally, cell proliferation was also estimated as an average of Ki-67-positive nuclei in relation to the total number of nuclei per microscopic field as previously reported [19]. To this end, $5 \times 10^{3}$ stably transfected cells were plated in $\mu$-Dish $35 \mathrm{~mm}$ from Ibidi.

\section{Cell adhesion assay}

The adhesion capacity of the breast cancer lines 4T1 and MDA-MB-231 was analysed using 24-cell culture plates (VWR) coated with fibronectin, laminin, type IV collagen (Sigma-Aldrich) and $\alpha_{v} \beta_{3}$ and $\alpha_{5} \beta_{1}$ integrins (R\&D Systems). Uncoated wells were employed as a control. For all conditions $3 \times 10^{5} \mathrm{cells} / \mathrm{mL}$ were employed, with the exception of MDA-MB -231 in the wells coated with integrin $\alpha_{v} \beta_{3}$ which were used at $1 \times 10^{4}$ cells $/ \mathrm{mL}$. Cells were seeded and allowed to adhere at $37{ }^{\circ} \mathrm{C}$ and $5 \% \mathrm{CO}_{2}$ for $30 \mathrm{~min}$. Then, non-adhered cells were removed by washing three times with PBS and remaining cells were fixed with 


\section{Cellular Physiology Cell Physiol Biochem 2019;53:87-100 \\ and Biochemistry Published online: 18 June $2019 \quad$\begin{tabular}{l|l} 
DOI: $10.33594 / 000000123$ & 2019 The Author(s). Published by \\
Cell Physiol Biochem Press GmbH 8 Co.
\end{tabular} \\ Mohamedi et al.: Fibulin-5 RGD Motif in Breast Cancer Cells}

4\% paraformaldehyde and stained with cell stain solution. All data are the mean of three independent experiments.

Invasion assay

In vitro invasion potential was evaluated using 24-well Matrigel-coated invasion chambers with a 8 $\mu \mathrm{m}$ pore size (BD Biosciences). To this end, $5 \times 10^{4} 4 \mathrm{~T} 1$ or MDA-MB- 231 cells were allowed to migrate for $24 \mathrm{~h}$ using $10 \%$ fetal bovine serum as a chemoattractant. At least three independent experiments were made for each condition. Non-invading cells on the upper surface were removed from the chambers using a cotton swab and cells that reached the lower surface were fixed with $4 \%$ paraformaldehyde and stained with crystal violet. Cells were counted in three randomly selected microscopic fields.

\section{Mammospheres culture}

To evaluate mammosphere formation capacity of breast tumor cells, $4 \times 10^{4} 4 \mathrm{~T} 1$ cells were plated in 6-well ultralow attachments plates (Corning Costar) and grown in MammoCult Basal Medium (Stemcell Technologies) supplemented with $10 \%$ MammoCult proliferation supplement, $4 \mu \mathrm{g} / \mathrm{mL}$ heparin and $0.5 \mu \mathrm{g} / \mathrm{mL}$ hydrocortisone. After 7 days, mammospheres were collected and enzymatically dissociated. Individual dissociated cells were cultured in 96-well ultralow attachment plates at a density of 20 cells/well. Mammosphere formation was monitored microscopically daily to ensure they derived from single cells and not from aggregates. Number of mammospheres was quantified and graphically represented after 7 days.

\section{Animals and subcutaneous tumors}

Six to eight-week-old male BALB/c mice (Charles River Laboratories) were used for in vivo studies. To induce subcutaneous tumors, four groups of five mice were injected at one flank with $1 \times 10^{5}$ cells of 4T1 Fibulin5-KD, 4T1 Fibulin5-RGE cells and 4T1 cells in $100 \mu \mathrm{L}$ PBS respectively. Tumor growth was monitored weekly and was measured with a calliper, and tumour volume was determined using the formula: $V=0.4$ $\times A \times B^{2}$, where $A$ is the largest dimension of the tumour and $B$ is the smallest dimension.

\section{Statistical analysis}

Data were analysed using GraphPad Prism 5.0 Software and represented as mean \pm SD, or mean \pm SE when indicated. Significant differences were determined with the Student $t$-test for parametric data and the Mann-Whitney test for non-parametric data. P-values $<0.05$ were considered statistically significant $\left({ }^{*} \mathrm{P}<0.05,{ }^{* *} \mathrm{P}<0.01,{ }^{* * *} \mathrm{P}<0.005\right)$.

\section{Results}

Fibulin-5RGE induces morphological changes in the $4 T 1$ murine breast cancer cell line

Murine cell line 4T1 is an experimental model for mammary human cancer [21]. To examine the influence of the fibulin-5 RGD motif in the behavior of 4T1 cells, we carried out site-directed mutagenesis to introduce the Asp to Glu change (RGD-to-RGE) at position 56 of the amino acid sequence of the human fibulin-5 (Fig. 1A). Following transfection, we selected clones containing the RGD-to-RGE change (4T1 Fibulin-5RGE cells). As a control, we used same cell line that exogenously expressed wild-type fibulin-5 (4T1 Fibulin-5WT cells). In addition, we carried out RNA interference on FBLN5 gene (4T1 Fibulin-5KD cells) for comparative purposes. Expression level of fibulin-5, Fibulin-5RGE and fibulin-5KD in selected clones was analyzed by Western-blot. 4T1 cells transfected with an empty vector were employed as control (Fig. 1B). Cell morphology was further examined with the finding that both 4T1 Fibulin-5RGE and 4T1 Fibulin-5KD cells shifted the epithelial-like phenotype of this cell line towards a mesenchymal-like phenotype (Fig. 1C). However, 4T1 Fibulin-5WT cells did not undergo any apparent morphological alteration. To investigate the mechanisms underlying those morphological changes, we explored the expression levels of E-cadherin, $\mathrm{N}$-cadherin and vimentin. Both 4T1 Fibulin-5RGE and 4T1 Fibulin-5KD cells showed a drastic decrease in E-cadherin expression with respect to control cells (Fig. 1D). By contrast, expression levels of N-cadherin and vimentin were increased in Fibulin-5RGE and 4T1 
A

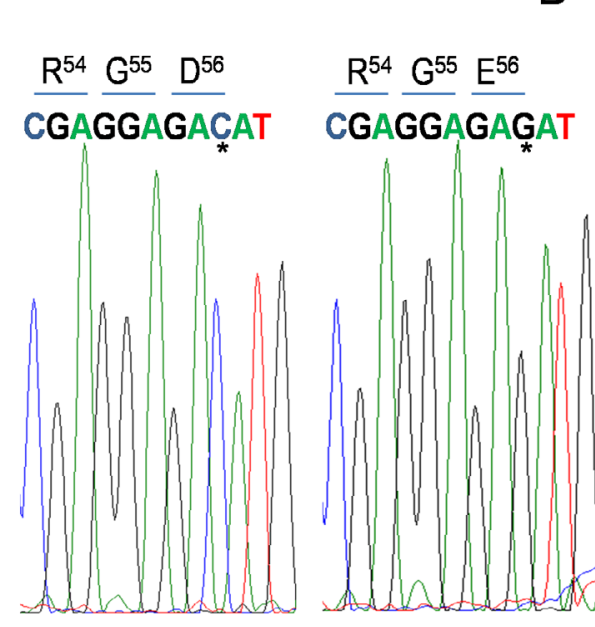

C

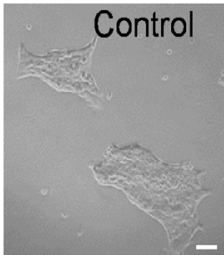

Fibulin-5WT

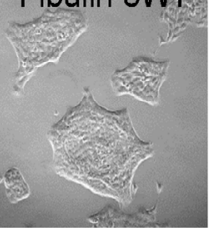

D

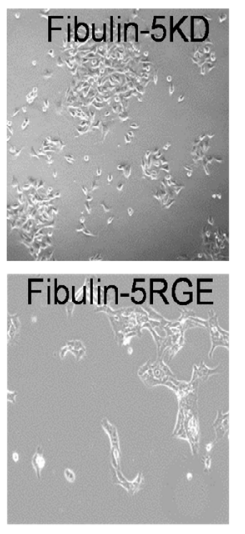

B<smiles>CC1C[C@H]2CO[C@H](C1)C2</smiles>

Fibulin-5 $=40 \mathrm{kDa}$ $\beta$-actin
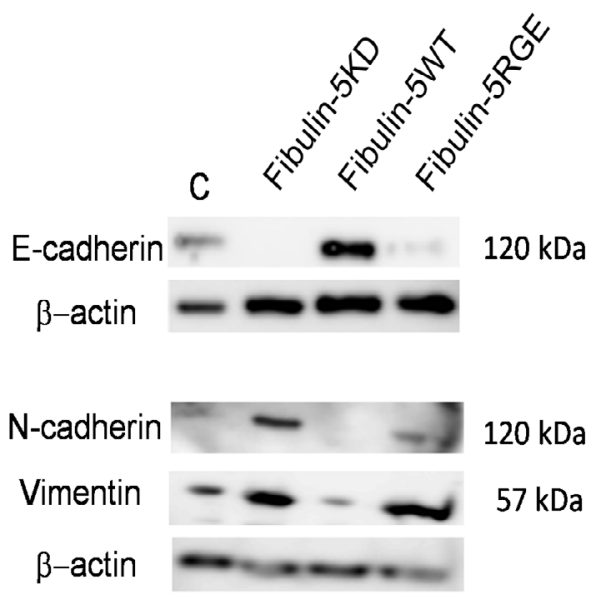

Fig. 1. Effects of the RGD-to-RGE in fibulin-5 in 4T1 cells. A. Sequencing electropherograms showing the substitution of $C$ to $G$ in the cDNA (indicated with an asterisk), resulting in a change D56E in the amino acid sequence of fibulin-5. B. Western-blot showing the expression of fibulin-5 in 4T1 cells. Control cells transfected with an empty vector are indicated as C, interfered cells as Fibulin-5KD, transfected with the full-cDNA for fibulin-5 as Fibulin-5WT, and cells transfected with cDNA for fibulin-5 containing the RGDto-RGE are indicated as Fibulin-5 RGE. Molecular weight is indicated on the right. $\beta$-actin was employed as loading control. Intervening irrelevant lanes are not shown. C. Morphological changes in fibulin-5 (Fibulin5KD) interfered 4T1 cells and in 4T1 cells containing the RGD-to-RGE change in fibulin-5. Bar, $100 \mu \mathrm{m}$. D. Western-blot showing the expression of the EMT markers E-cadherin, N-cadherin and vimentin in the indicated cells. Molecular weight markers are indicated on the right and $\beta$-actin was employed as loading control.

Fibulin-5KD cells, but decreased in Fibulin-5WT cells. Overall, these data are compatible with an alteration of the epithetial-to-mesenchymal transtition (EMT) process due to the RGD-to-RGE change in fibulin-5 when expressed in 4T1 cells.

\section{Fibulin-5RGE increases proliferation of 4 T1 cells}

We have previously shown that presence of exogenous fibulin-5 reduces proliferation of human breast cancer cells which do not express endogenous fibulin-5 [19]. Now, we wanted to evaluate whether the proliferation of the murine 4T1 cell line, which endogenously 
A
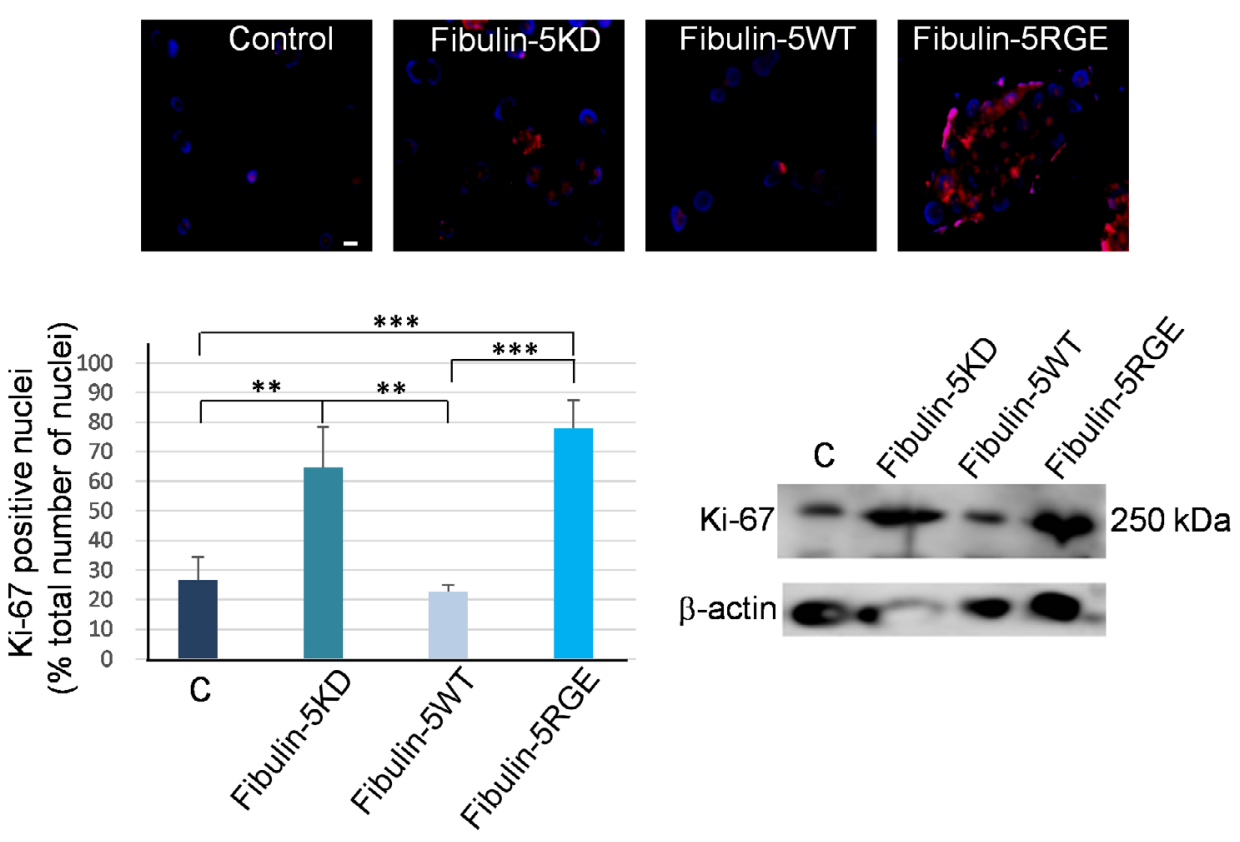

B

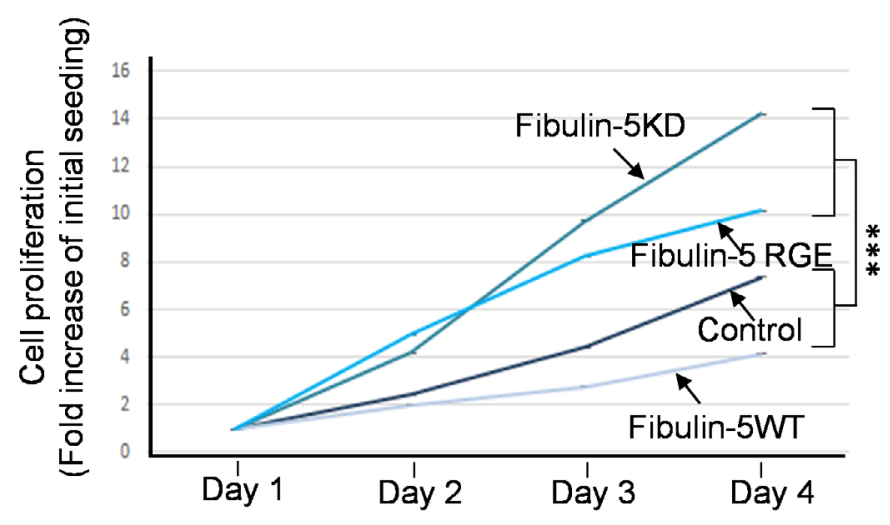

Fig. 2. RGD-to-RGE change in fibulin-5 affects proliferation of $4 \mathrm{~T} 1$ cells. A. Expression of the proliferative nuclear marker Ki-67 was examined by immunocytochemistry. Top, representative images of Ki-67 staining (in red) in the indicated 4T1 cells. Cell nuclei are shown in blue; bar, $10 \mu \mathrm{m}$. Bottom left, quantification of Ki-67 positive nuclei. Bottom right, Western-blot analysis showing Ki-67 expression in the indicated cells. Molecular weight markers are indicated on the right and $\beta$-actin was employed as loading control. B. Cell proliferation of the 4T1 cells employed in this work measured by MTT assay.

expresses fibulin-5, can be affected by the exogenous expression of fibulin-5 or fibulin5-RGE. To this end we performed two different approaches. First we examined expression of the nuclear marker Ki-67 (Fig. 2A). Number of Ki-67 positive nuclei does not differ substantially between control (an average of $27 \%$ of cells showed Ki-67-positive nuclei staining) and 4T1 Fibulin-5WT cells (23\%). However, 4T1 Fibulin-5RGE cells showed considerable increased in the number of Ki-67 positive nuclei (78\%) respecting control and 4T1 Fibulin-5WT cells. Interestingly, 4T1 Fibulin-5KD cells also increased number of positive Ki-67 nuclei (65\%). Differences in Ki-67 expression could be also appreciated by Western-blot (Fig. 2A). 
Second approach consisted of a MTT assay (Fig. 2B). Again 4T1 Fibulin-5RGE and 4T1 Fibulin-5KD cells showed an increased level of proliferation respect to control and 4T1 Fibulin5WT cells. These results illustrate the influence of an intact fibulin-5 to control breast cancer cell proliferation.

Fibulin-5RGE increases the capacity of $4 \mathrm{T1}$ cells to form mammospheres 4T1 cells shows a strong capacity to form mammopheres [22]. To examine whether the exogenous expression of Fibulin-5RGE could affect this capacity, mammosphere-forming units (MFU) were counted for the different conditions assayed. As can be seen in Fig. 3, 4T1 Fibulin5RGE cells showed the highest capacity to form mammospheres in two consecutive passages. Thus, in the first passage 4T1 Fibulin-5RGE cells showed a 3.2-fold increase in their ability to form mammospheres with respect to control cells; and 2-fold increase in the second passage. This ability is also increased in 4T1 Fibulin-5KD cells, but only in the first passage (2-fold increase). We also noticed that size of

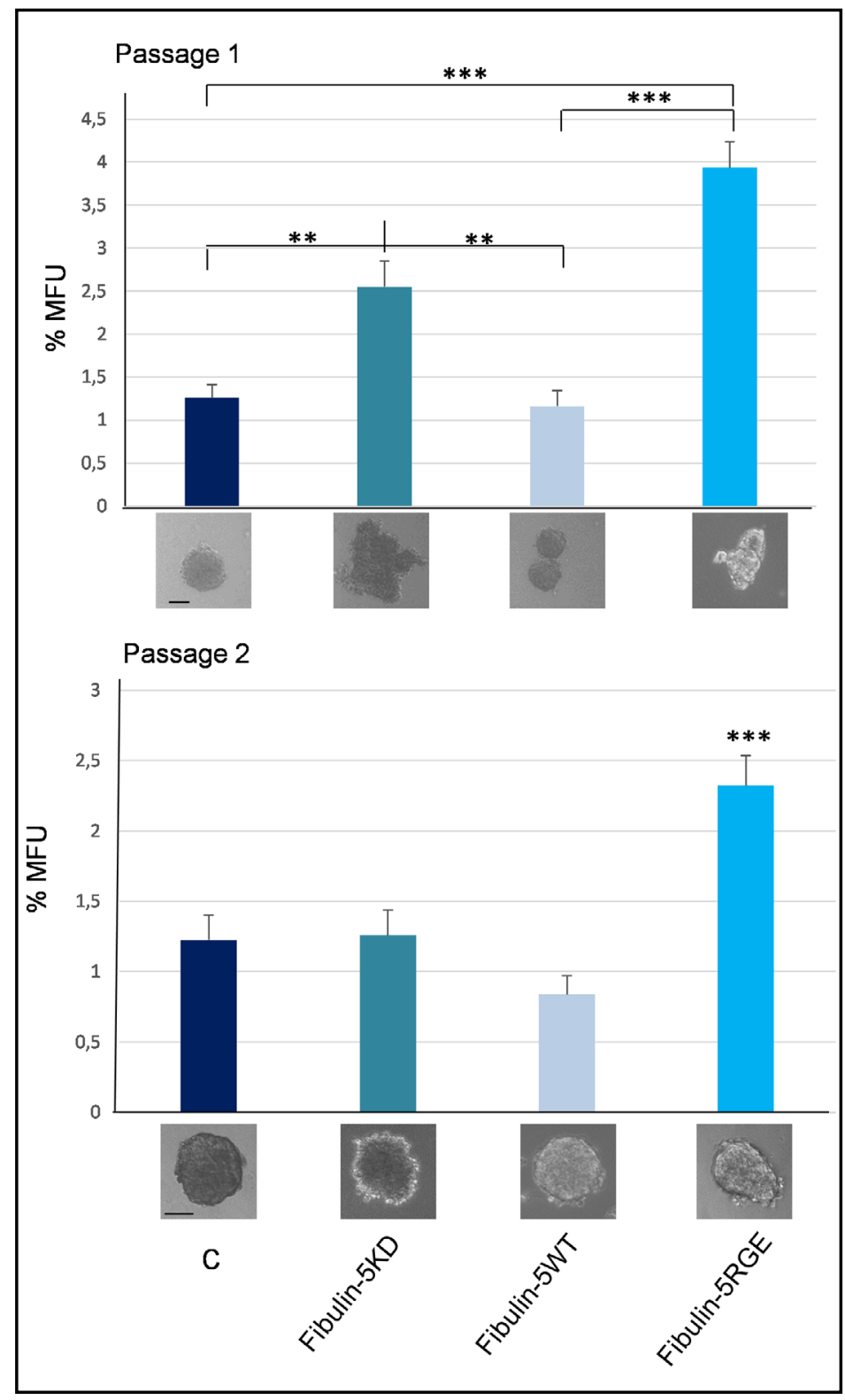

Fig. 3. Mammosphere-formation assay using $4 \mathrm{~T} 1$ cells. Quantification (mammosphere-forming unit, MFU, calculated as a percentage of mammospheres formed from the number of cells seeded). Bars, standard error. Representative images of mammospheres derived from the cells employed in this work in two consecutive passages are also shown. Morphological changes are mainly observed in 4T1 Fibulin5KD and 4T1 Fibulin-5RGE cells. Bar, $50 \mu \mathrm{m}$. mammospheres did not show significant changes among the conditions assayed. However, sharp morphological changes can be observed in mammospheres derived from 4T1 Fibulin-5RGE cells and 4T1 Fibulin-5KD cells (Fig. 3). These changes can be related with the decrease in $\mathrm{N}$-cadherin expression [23], and it could indicate that the anchorage-independent growth may be facilitated in breast cancer cells harboring the RGD-to-RGE change in fibulin-5. 


\section{Cellular Physiology Cell Physiol Biochem 2019;53:87-100

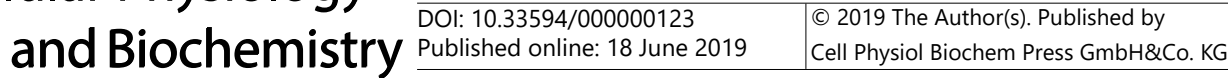 \\ Mohamedi et al.: Fibulin-5 RGD Motif in Breast Cancer Cells}

Influence of the RGD-to-RGE change in fibulin-5 in the adhesion and invasion properties of 4 T1 cells

To explore whether the RGD-to-RGE change in fibulin-5 may alter adhesion properties of 4T1 cells, we analyzed the capacity of the cells to bind to the ECM components fibronectin, laminin and type IV collagen. Results indicated that 4T1 Fibulin-5WT cells showed the highest capacity to bind to culture plates coated with these ECM components but also the binding to standard culture plates (Fig. 4A). In fact, above 180 cells per field were counted in the cases of binding of 4T1 Fibulin-5WT cells to fibronectin and type IV collagen, and it is noteworthy that in the case of fibronectin the number of attached cells was closed to 250 per field. On the contrary, number of attached 4T1 Fibulin-5KD and 4T1 Fibulin-5RGE cells was between 50 and 75 cells per field in all conditions assayed, including standard culture plates.

We also wanted to know the capacity of 4T1 cells to bind to culture plates coated with $\alpha_{v} \beta_{3}$ and $\alpha_{5} \beta_{1}$ integrins (Fig. 4B). Again 4T1 Fibulin-5WT cells showed the highest capacity of attachment to both integrins (58 in the case of $\alpha_{v} \beta_{3}$ and and 48 in the case of $\alpha_{5} \beta_{1}$ cells per field respectively), followed by control cells ( 42 and 36 cells per field). By contrast, neither 4T1 Fibulin-5KD (11 and 19 cells per field) nor 4T1 Fibulin-5RGE (14 and 22 cells per field) cells showed a strong capacity to bind to the integrins $\alpha_{v} \beta_{3}$ or $\alpha_{5} \beta_{1}$.

To examine the influence of the RGD-to-RGE change in fibulin-5 on the invasive capacity of 4T1 cells, we employed transwell inserts coated with Matrigel (Fig. 4C). Both 4T1 Fibulin5 KD cells (99 cells per field) and 4T1 Fibulin-5RGE cells (79 cells per field) showed an increase in their invasive properties as compared to $4 \mathrm{~T} 1$ control cells (52 cells per field). In this assay, 4T1 Fibulin-5WT cells showed the lowest capacity to invade (28 cells per field). These data strongly suggest the influence of fibulin- 5 in both cell attachment and cell invasion processes.

\section{RGD-to-RGE change in fibulin-5 potentiates capacity of $4 T 1$ cell to form tumors}

The effect of the RGD-to-RGE change in fibulin-5 was examined in vivo through the subcutaneous injection of 4T1 Fibulin-5RGE cells in the flank of five male BALB/c mice. 4T1 Fibulin-5, 4T1 Fibulin-5KD cells were employed for comparative analysis. Five mice were injected with 4T1 cells transfected with an empty vector as control purposes. Volumes of subcutaneous tumors formed were measured for six weeks (Fig. 5A). Tumors derived from 4T1 Fibulin-5KD cells, but especially from 4T1 Fibulin-5RGE cells, showed a considerable larger volumes than those derived for control cells and tumors were macroscopically visible at three weeks. These results reinforce the tumor-protective effect of fibulin-5, but also highlight the involvement of its RGD domain to induce these effects. In addition, we have also verified levels of fibulin-5 expression in tumors derived from Fibulin-5KD 4T1, Fibulin5RGE and control 4T1 cells (Fig. 5B).

\section{RGD-to-RGE change in fibulin-5 alters behavior of MDA-MB-231 cells}

Next, we wanted to know whether the results described above could be reproduced in a breast cancer cell which does not express fibulin-5 endogenously. To this end we employed the MDA-MB-231 cell line due to the expression level of fibulin-5 is undetectable by Westernblot [19]. Following selection of cells expressing fibulin-5RGE (MDA-MB-231 Fibulin5RGE) and wild-type fibulin-5 (MDA-MB-231 Fibulin-5WT) (Fig. 6A), we first examined the expression of the EMT markers E-cadherin, N-cadherin and vimentin. Main findings consisted of an increase of E-cadherin expression level but a decrease of $\mathrm{N}$-cadherin and vimentin expression levels in MDA-MB-231 Fibulin-5WT cells with respect to both MDAMB-231 Fibulin-5RGE cells and MDA-MB-231 control cells. Then, we evaluated the ability of the MDA-MB-231 to adhere to the ECM components fibronectin, laminin and type IV collagen. Results revealed that MDA-MB-231 Fibulin-5WT cells showed a high capacity to bind to fibronectin (144 cells per field), laminin (114 cells per field) and type IV collagen (113 cells per field), as compared with Fibulin-5RGE cells (15 to laminin and 19 to type IV collagen cells per field respectively) and control cells (23 to laminin and 15 to type IV collagen, Fig. 6B). In addition, we also examined the binding of those cells to wells coated with $\alpha_{v} \beta_{3}$ integrin. MDA- 


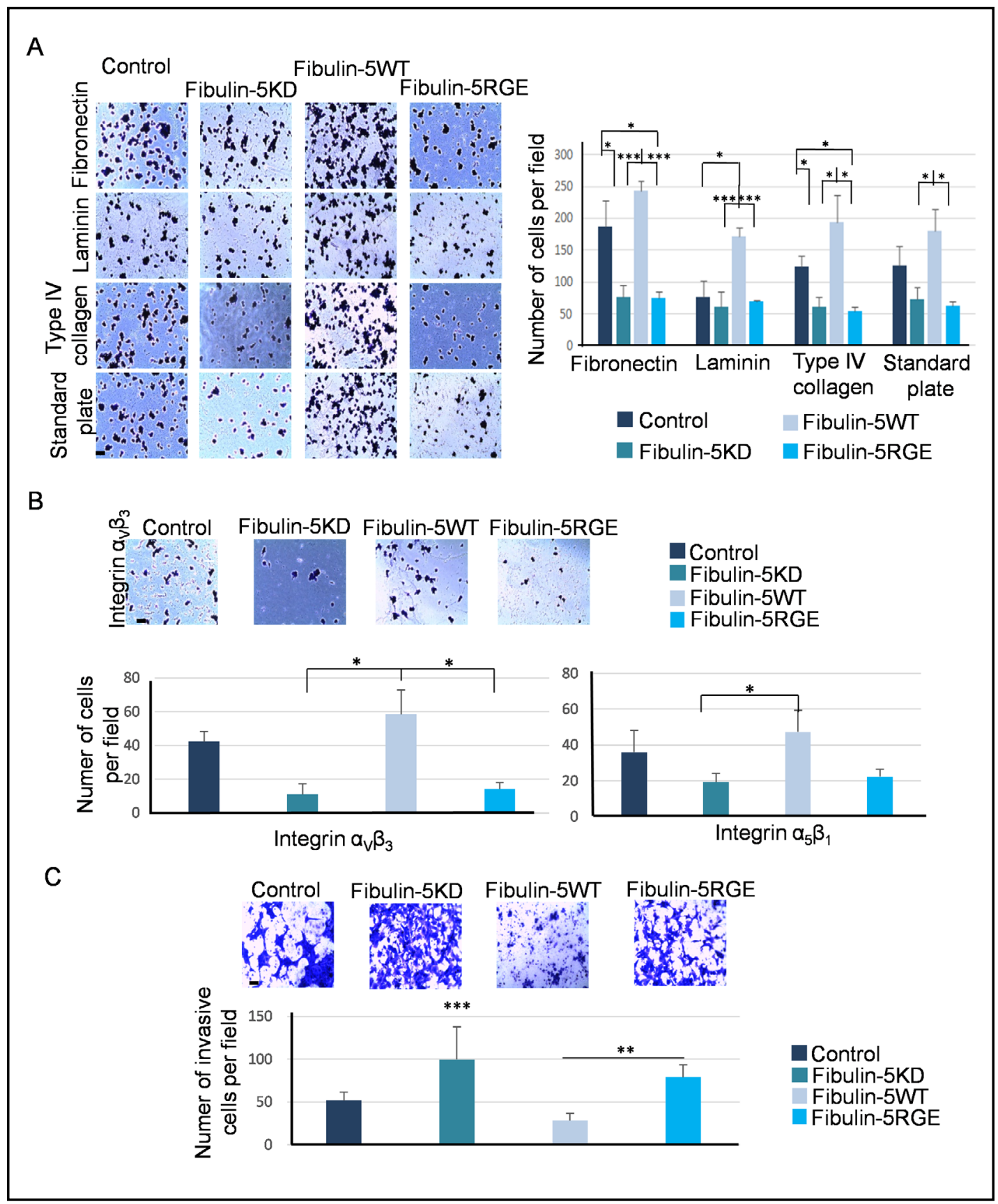

Fig. 4. RGD-to-RGE change in fibulin-5 alters the adhesion profile and the invasive ability of $4 \mathrm{~T} 1$ cells. A. Left, representative images showing attachment of the indicated 4T1 cells to the ECM components fibronectin, laminin and type IV collagen. Adhesion to standard culture plates was also examined. Bar, 10 $\mu \mathrm{m}$. Right, graphical representation of the number of attached cells. Exogenous expression of an intact fibulin-5 (Fibulin-5WT) increases the number of attached 4T1 cells in all assayed conditions. B. Ability of the $4 \mathrm{~T} 1$ cells used in this work to bind to $\alpha \alpha_{v} \beta_{3}$ integrin was also examined. Top, representative images of the indicated $4 \mathrm{~T} 1$ cells attached to the $\alpha_{\mathrm{v}} \beta_{3}$ integrin. Bar, $10 \mu \mathrm{m}$. Bottom, graphical representation of the number of attached cells to the $\alpha_{v} \beta_{3}$ and $\alpha_{5} \beta_{1}$ integrins as indicated. Exogenous expression of an intact fibulin-5 (Fibulin-5WT) also increases adhesion of 4 T1 cells (color code as in C) to the $\alpha_{v} \beta_{3}$ integrin. C. Top, representative pictures showing invasive 4T1 cells as indicated using Matrigel-coated invasion chambers. Bar, $10 \mu \mathrm{m}$. Bottom, quantification of the number of invasive cells. 
A

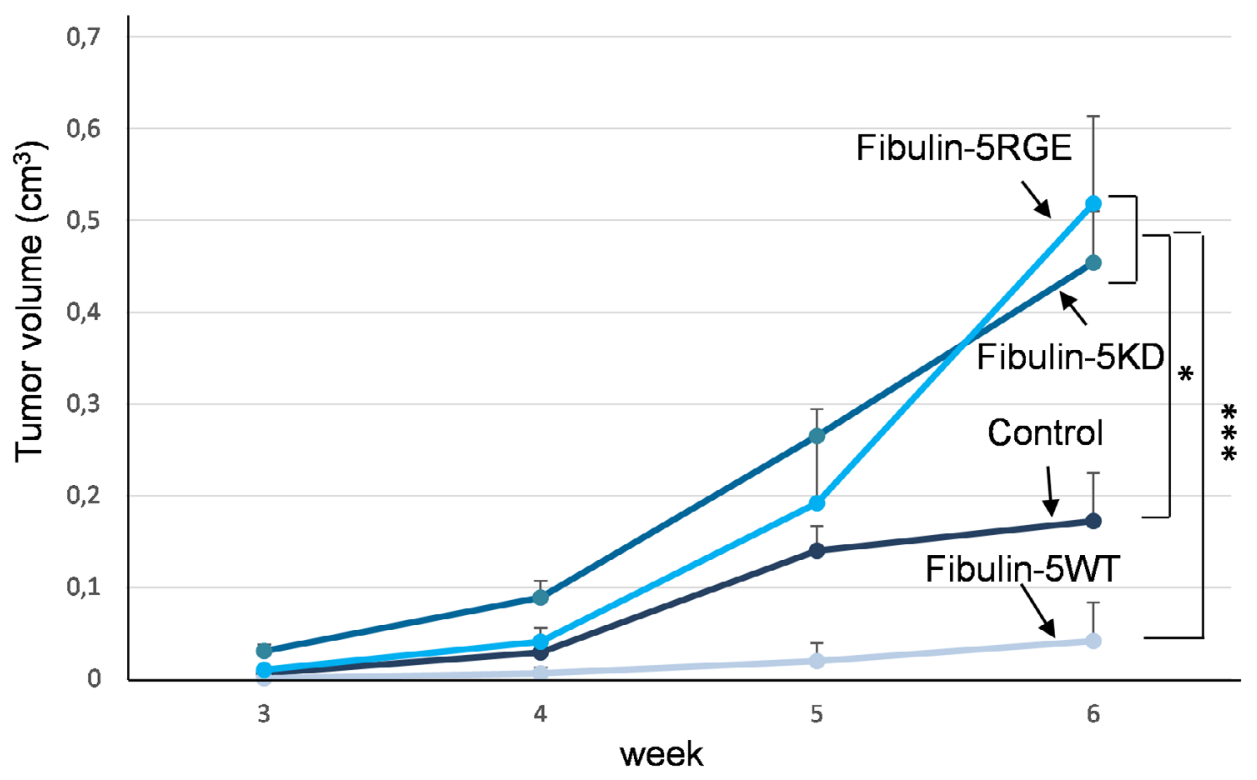

$\mathrm{B}$ Fibulin-5KD Fibulin-5RGE Control

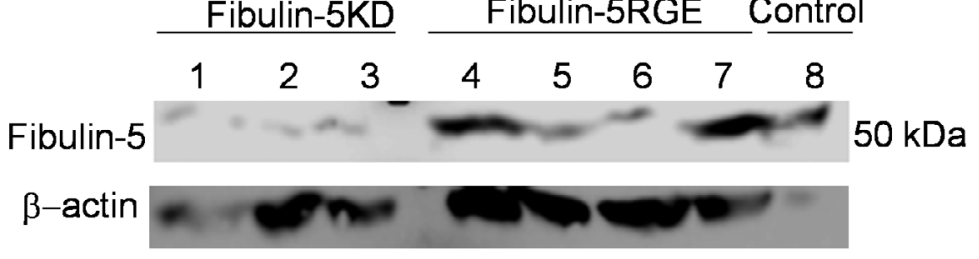

Fig. 5. RGD-to-RGE change in fibulin- 5 increases the capacity of $4 \mathrm{~T} 1$ cells to form subcutaneous tumors. Top, graphical representation of the tumor volumes formed by the indicated 4T1 cells when injected in the flank of BALB/c mice. Tumor volumes were measured for six weeks. Bottom, western-blot analysis of the fibulin-5 expression in three tumors formed by 4T1 Fibulin-5KD cells, four 4T1 Fibulin-5RGE cells and one control cells. Molecular weight marker is indicated on the right and $\beta$-actin was employed as loading control.

MB-231 Fibulin-5WT also increased their capacity of binding to this integrin (18 cells per field) as compared to both control ( $\sim 3$ cells per field) and MDA-MB-231 Fibulin-5RGE cells ( $\sim 4$ cells per field, Fig. $6 \mathrm{C}$ ). Other results supporting the effect of the RGD-to-RGE change in MDA-MB-231 cells are indicated in Supplementary Fig. 1 and Supplementary Fig. 2 (for all supplemental material see www.cellphysiolbiochem.com). Thus, we performed an invasion assay to examine whether the differences in binding could influence invasive capacity of this cell line. Invasion ability of MDA-MB-231 cells was considerable reduced when expressing exogenous fibulin-5 (MDA-MB-231 Fibulin-5WT, 60 cells per field, Supplementary Fig. 1), as compared to invasion capacities of control (140 cells per field) and MDA-MB-231 Fibulin5RGE cells (188 cells per field). We also wanted to examine if Fibulin-5RGE induces similar effects on MDA-MB-231 cells proliferation than those described above for 4 T1 cells. A MTT analysis revealed that fibulin-5 does not alter capacity of MDA-MD-231 cells to proliferate, contrary to what was observed for MDA-MB-231 Fibulin-5WT cells (Supplementary Fig. 2). Taken together, these results strongly suggest that changes in the RGD motif of fibulin-5 may induce changes in breast cancer cells that ultimately lead to increase their protumor properties. 


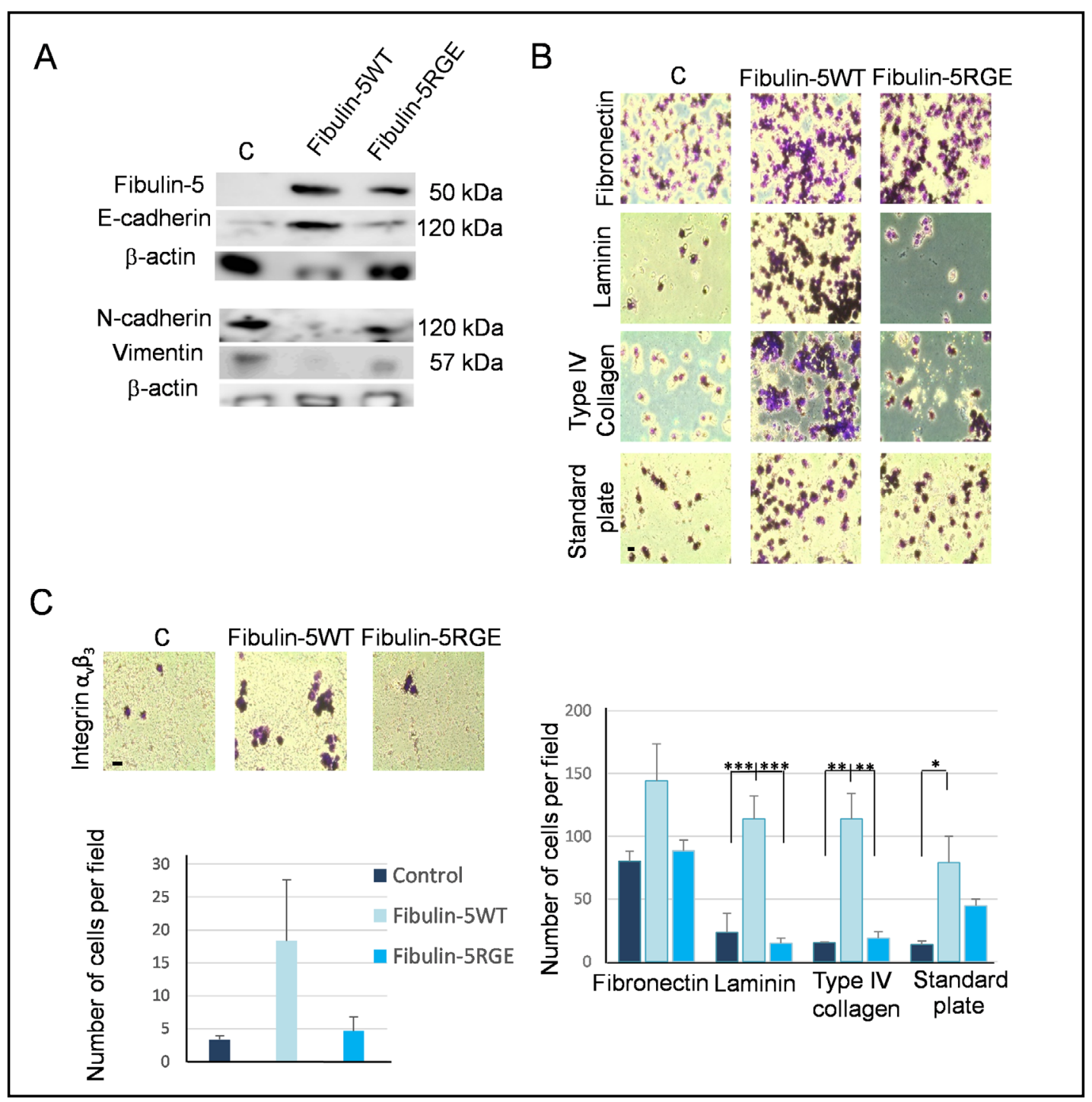

Fig. 6. RGD-to-RGE change in fibulin-5 modifies behavior of MD-MB-231 cells. A. Western-blot showing the expression of the EMT markers E-cadherin, N-cadherin and vimentin in control MDA-MB-231 cells (C), MDA-MB-231 cells tranfected with the full-length cDNA for fibulin-5 (Fibulin-5WT), or transfected with the cDNA carrying the mutation to produce fibulin-5 containing the D56E change within its RGD motif (Fibulin5RGE). Molecular weight markers are indicated on the right and $\beta$-actin was employed as loading control. B. Top, representative images showing attachment of the indicated MDA-MB-231 cells to the ECM components fibronectin, laminin and type IV collagen. Adhesion to standard culture plates was also examined. Bar, 10 $\mu \mathrm{m}$. Bottom, graphical representation of the number of attached cells. C. Ability of the MDA-MB-231 cells used in this work to bind to the $\alpha_{v} \beta_{3}$ integrin was also examined. Top, representative images of the indicated MDA-MB-231 cells attached to the $\alpha_{v} \beta_{3}$ integrin. Bar, $10 \mu \mathrm{m}$. Bottom, graphical representation of the number of attached cells as indicated. 


\section{Cellular Physiology Cell Physiol Biochem 2019;53:87-100 \\ \begin{tabular}{ll|l} 
and Biochemisty $10.33594 / 000000123$ & C 2019 The Author(s). Published by \\
Published online: 18 June 2019 & Cell Physiol Biochem Press GmbH\&Co. KG
\end{tabular} \\ Mohamedi et al.: Fibulin-5 RGD Motif in Breast Cancer Cells}

\section{Discussion}

Fibulin-5 is a structurally complex extracellular protein. Within its N-terminal region, six calcium-binding epithelial growth factor-like (cbEGF-like) domains can be identified and, in turn, a RGD motif is located in the first cbEGF-like domain [7]. It has been largely proven the function of the RGD motif in cell adhesion as well as its potential application in tumor therapy [24]. In the case of fibulin-5, it is known that its RGD motif mediates cell binding through the interaction with different integrins including $\alpha_{v} \beta_{3,} \alpha_{v} \beta_{5}, \alpha_{5} \beta_{1}$ or $\alpha_{4} \beta 1[9,25]$. For instance, fibulin-5 RGD motif can influence the attachment and spreading of primary aortic smooth muscle cells through $\beta_{1}$ integrins. However, the interaction between fibulin-5 RGD motif and $\beta_{1}$ integrins failed to activate integrin-mediated signaling pathways, suggesting that fibulin-5 may act as a blocking factor [25].

In a tumor context, it has been shown that loss of interaction between fibulin- 5 and $\beta_{1}$ integrins hinders tumor growth by increasing the level of reactive oxygen species, affecting the angiogenesis process [20]. However, different reports have highlighted the tumor suppressor functions of fibulin-5 in bladder or lung cancers $[5,12,13]$. We have also previously shown that the exogenous expression of fibulin- 5 also confers antitumor properties to breast cancer cell lines [19]. In this work we wanted to explore whether the RGD motif could affect the antitumor role of fibulin-5 in breast cancer cells. To this end we exogenously expressed fibulin-5 containing the RGD-to-RGE change in the murine 4T1 and the human MDA-MB-231 breast cancer cell lines. Different cell-based assays have revealed that the RGD-to-RGE change drastically abolishes the antitumor effect that an intact fibulin-5 displays, and similar results were obtained when we performed knock-down of the FBLN5 gene. Overall these data suggest that the RGD-to-RGE change induce effects comparable to those derived from a loss of function of the FBLN5 gene. In line with these results, a previous report has shown that fibulin-5 promotes adhesion to fibronectin and reduces invasion of hepatocellular carcinoma cells by an integrin-dependent mechanism [26]. However, these effects were not observed when the RGD motif of fibulin-5 was changed to RGE [26]. We also wanted to examine whether the RGD motif of fibulin-5 affects the binging of 4T1 and MDAMB-231 breast cancer cells to different ECM components as well as the $\alpha_{v} \beta_{3}$ integrin. Results obtained with MDA-MB-231 cells, which endogenous expression of fibulin-5 is absent or scarcely detected [19], revealed that an intact RGD motif increases the binding of MDAMB-231 cells particularly to the basal lamina components laminin and type IV collagen, as well as to the $\alpha_{v} \beta_{3}$ integrin. In stark contrast, the RGD-to-RGE change drastically reduces this binding. Previous reports have involved $\alpha_{v} \beta_{3}$ integrin in breast cancer. Thus, $\alpha_{v} \beta_{3}$ integrin increases metastasis of breast cancer cells to bone [27], therefore it could be a therapeutic target for prevention of skeletal metastasis [28]. Moreover, MDA-MB-231 cells expressing fibulin-5 harboring the RGD-to-RGE change do not reduce their ability to invade. Thus, it could be speculated that the RGD motif is a key structural element to promote the antitumor effects of fibulin-5, which could be increased by blocking the signaling pathways mediated by the activation of integrins that could interact with fibulin-5. These findings would reinforce the antitumor effects that intact fibulin- 5 can display within the tumor microenvironment.

\section{Conclusion}

Our results provide new evidence about the relevance of fibulin-5 in proliferation, invasion and the induction of EMT process in 4T1breast cancer cells. Induction of cell proliferation and EMT have been also described due to different factors in other commonly employed breast cancer cell lines [29,30]. Our data also highlight the context-specific effects associated to fibulin-5 [11,31]. These findings should help to decipher some of the molecular mechanisms underlying the tumor-protecting functions elicited by fibulin-5. Also, our findings deepen on the tumor-protective functions that an intact fibulin-5 induces in the 


\section{Cellular Physiology Cell Physiol Biochem 2019;53:87-100

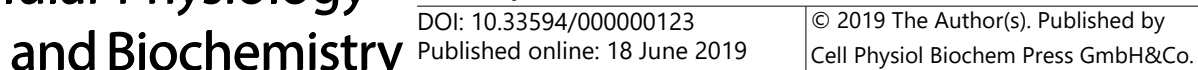 \\ Mohamedi et al.: Fibulin-5 RGD Motif in Breast Cancer Cells}

tumor microenvironment, which could contribute the development of future therapies for breast cancer treatment.

\section{Acknowledgements}

This work was supported by the Instituto Asturiano de Odontología (IAO). Yamina Mohamedi is recipient of a fellowship from the FICYT, Fundación para el Fomento en Asturias de la Investigación Científica Aplicada y la Tecnología ("Severo Ochoa" Research Program, Principado de Asturias), and Tania Fontanil is recipient of a contract from the IAO. Mice were housed under specific pathogen-free conditions and following the guidelines of the Committee on Animal Experimentation of the Universidad de Oviedo, Asturias, Spain.

\section{Disclosure Statement}

The authors declare no conflicts of interest.

\section{References}

1 Bonnans C, Chou J, Werb Z: Remodelling the extracellular matrix in development and disease. Nat Rev Mol Cell Biol 2014;15:786-801.

2 Rozario T, DeSimone DW: The extracellular matrix in development and morphogenesis: a dynamic view. Dev Biol 2010;341:126-140.

3 Lu P, Weaver VM, Werb Z: The extracellular matrix: a dynamic niche in cancer progression. J Cell Biol 2012;196:395-406.

$4 \quad$ Gallagher WM, Currid CA, Whelan LC: Fibulins and cancer: friend or foe? Trends Mol Med 2005;11:336340.

5 Obaya AJ, Rua S, Moncada-Pazos A, Cal S: The dual role of fibulins in tumorigenesis. Cancer Lett 2012;325:132-138.

6 de Vega S, Iwamoto T, Yamada Y: Fibulins: multiple roles in matrix structures and tissue functions. Cell Mol Life Sci 2009;66:1890-1902.

7 Yanagisawa H, Schluterman MK, Brekken RA: Fibulin-5, an integrin-binding matricellular protein: its function in development and disease. J Cell Commun Signal 2009;3:337-347.

8 Papke CL, Yanagisawa H: Fibulin-4 and fibulin-5 in elastogenesis and beyond: Insights from mouse and human studies. Matrix Biol 2014;37:142-149.

9 Nakamura T, Lozano PR, Ikeda Y, Iwanaga Y, Hinek A, Minamisawa S, Cheng CF, Kobuke K, Dalton N, Takada Y, Tashiro K, Ross Jr J, Honjo T, Chien KR: Fibulin-5/DANCE is essential for elastogenesis in vivo. Nature 2002;415:171-175.

10 Yanagisawa H, Davis EC, Starcher BC, Ouchi T, Yanagisawa M, Richardson JA, Olson EN: Fibulin-5 is an elastin-binding protein essential for elastic fibre development in vivo. Nature 2002;415:168-171.

11 Schiemann WP, Blobe GC, Kalume DE, Pandey A, Lodish HF: Context-specific effects of fibulin-5 (DANCE/ EVEC) on cell proliferation, motility, and invasion. Fibulin-5 is induced by transforming growth factor-beta and affects protein kinase cascades. J Biol Chem 2002;277:27367-27377.

12 Hu Z, Ai Q Xu H, Ma X, Li HZ, Shi TP, Wang C, Gong DJ, Zhang X: Fibulin-5 is down-regulated in urothelial carcinoma of bladder and inhibits growth and invasion of human bladder cancer cell line 5637 . Urol Oncol 2011;29:430-435.

13 Yue W, Sun Q, Landreneau R, Wu C, Siegfried JM, Yu J, Zhang L: Fibulin-5 suppresses lung cancer invasion by inhibiting matrix metalloproteinase-7 expression. Cancer Res 2009;69:6339-6346.

14 Chen X, Song X, Yue W, Chen D, Yu J, Yao Z, Zhang L: Fibulin-5 inhibits Wnt/beta-catenin signaling in lung cancer. Oncotarget 2015;6:15022-15034. 


\section{Cellular Physiology Cell Physiol Biochem 2019;53:87-100

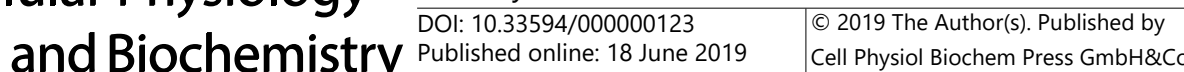 \\ Mohamedi et al.: Fibulin-5 RGD Motif in Breast Cancer Cells}

15 Ohara H, Akatsuka S, Nagai H, Liu YT, Jiang L, Okazaki Y, Yamashita Y, Nakamura T, Toyokuni S: Stagespecific roles of fibulin-5 during oxidative stress-induced renal carcinogenesis in rats. Free Radic Res 2011;45:211-220.

16 Hwang CF, Shiu LY, Su LJ, Yin YF, Wang WS, Huang SC, Chiu TJ, Huang CC, Zhen YY, Tsai HT, Fang FM, Huang TL, Chen CH: Oncogenic fibulin-5 promotes nasopharyngeal carcinoma cell metastasis through the FLJ10540/AKT pathway and correlates with poor prognosis. PLoS One 2013;8:e84218.

17 Lee YH, Albig AR, Regner M, Schiemann BJ, Schiemann WP: Fibulin-5 initiates epithelial-mesenchymal transition (EMT) and enhances EMT induced by TGF-beta in mammary epithelial cells via a MMPdependent mechanism. Carcinogenesis 2008;29:2243-2251.

18 Xiao W, Zhou S, Xu H, Li H, He G, Liu Y, Qi Y: Nogo-B promotes the epithelial-mesenchymal transition in HeLa cervical cancer cells via Fibulin-5. Oncol Rep 2013;29:109-116.

19 Mohamedi Y, Fontanil T, Solares L, Garcia-Suarez O, Garcia-Piqueras J, Vega JA, Cal S, Obaya AJ: Fibulin-5 downregulates Ki-67 and inhibits proliferation and invasion of breast cancer cells. Int J Oncol 2016;48:1447-1456.

20 Schluterman MK, Chapman SL, Korpanty G, Ozumi K, Fukai T, Yanagisawa H, Brekken RA: Loss of fibulin-5 binding to beta1 integrins inhibits tumor growth by increasing the level of ROS. Dis Model Mech 2010;3:333-342.

21 Pulaski BA, Ostrand-Rosenberg S: Mouse 4T1 breast tumor model. Curr Protoc Immunol 2001; DOI:10.1002/0471142735.im2002s39.

22 Wei L, Liu TT, Wang HH, Hong HM, Yu AL, Feng HP, Chang WW: Hsp27 participates in the maintenance of breast cancer stem cells through regulation of epithelial-mesenchymal transition and nuclear factorkappaB. Breast Cancer Res 2011;13:R101.

23 Manuel Iglesias J, Beloqui I, Garcia-Garcia F, Leis O, Vazquez-Martin A, Eguiara A, Cufi S, Pavon A, Menendez JA, Dopazo J, Martin AG: Mammosphere formation in breast carcinoma cell lines depends upon expression of E-cadherin. PloS One 2013;8:e77281.

24 Wang F, Li Y, Shen Y, Wang A, Wang S, Xie T: The functions and applications of RGD in tumor therapy and tissue engineering. Int J Mol Sci 2013;14:13447-13462.

25 Lomas AC, Mellody KT, Freeman LJ, Bax DV, Shuttleworth CA, Kielty CM: Fibulin-5 binds human smoothmuscle cells through alpha5beta1 and alpha4beta1 integrins, but does not support receptor activation. Biochem J 2007;405:417-428.

26 Tang JC, Liu JH, Liu XL, Liang X, Cai XJ: Effect of fibulin-5 on adhesion, migration and invasion of hepatocellular carcinoma cells via an integrin-dependent mechanism. World J Gastroenterol 2015;21:11127-11140.

27 Sloan EK, Pouliot N, Stanley KL, Chia J, Moseley JM, Hards DK, Anderson RL: Tumor-specific expression of alphavbeta3 integrin promotes spontaneous metastasis of breast cancer to bone. Breast Cancer Res 2006;8:R20.

28 Zhao Y, Bachelier R, Treilleux I, Pujuguet P, Peyruchaud O, Baron R, Clement-Lacroix P, Clezardin P: Tumor alphavbeta3 integrin is a therapeutic target for breast cancer bone metastases. Cancer Res 2007;67:58215830 .

29 Pei YF, Lei Y, Liu XQ: miR-29a promotes cell proliferation and EMT in breast cancer by targeting ten eleven translocation 1. Biochim Biophys Acta 2016;1862:2177-2185.

30 Jiang G, Shi W, Fang H, Zhang X: miR27a promotes human breast cancer cell migration by inducing EMT in a FBXW7dependent manner. Mol Med Rep 2018;18:5417-5426.

31 Albig AR, Schiemann WP: Fibulin-5 function during tumorigenesis. Future Oncol 2005;1:23-35. 